\title{
Measuring Technology of the Anisotropic Tensile Properties of Woven
}

\section{Fabrics}

Jiaming Zheng, Masayuki Takatera, Shigeru Inui, and Yoshio Shimizu

Faculty of Textile Science and Technology, Shinshu University, Ueda, Nagano, Japan

\begin{abstract}
A new creditable and effective multi-axial circular tensile tester (MACTT) with 16 load sensors installed around a circumference has been developed. The new tester can be used to measure tensile properties in various directions with one sample simultaneously under the same initial conditions. As a result, not only an error can be reduced but also the experiment samples and time can be saved. The experiment results show good agreement with the theoretically approximate values calculated based on the Hooke's Law. We have also compared the stress distributions obtained by the new tester with those tested by the KES (Kawabata's Evaluation System) instrument and the results confirm that the new tester is theoretically reasonable and practically feasible for measuring orthotropic tensile properties of the fabrics with symmetrical structures, and that the new tester can also be used to test and evaluate the anisotropic tensile properties of those fabrics with no orthogonal main axes.
\end{abstract}

\section{Introduction}

Although the applications of fabrics have expanded into various industrial areas, such as composite materials, buildings and machine elements, our understanding of the mechanical 
behavior of woven fabrics is still limited. Fabric properties are a mixture of the properties of the individual fiber and those of the structure of which they are constructed. It is well known that woven fabrics are typical anisotropic materials, especially; their tensile properties show remarkable anisotropic behavior.

The tensile properties of woven fabrics have been measured and studied by many researchers. As early as in 1937, Peirce [16] began this study from the viewpoint of the geometrical analysis of the weave structure. Chadwick et al. [5] presented a theoretical model, which was called "trellis model", to simulate the in-planar stress-train relationship of woven fabrics under a simple pull. One of the main defects of this model is that it does not exhibit the Poisson's effect, whereas real fabrics generally do. As a further work, Kilby [13] introduced the classical elasticity theory with the assumption of a fabric regarded as an anisotropic lamina, which showed the Poisson's effect and with two planes of symmetry at right-angles to one another. He also measured the tensile properties of woven fabrics in any direction using roller clamps method presented by Weissenberg et al. [19]. Kawabata et al. [8, 10-12] systematically carried out researches into the tensile properties of plain-weave fabrics and presented the tensile deformation theory based on fabric structural mechanics. Much useful research work over this topic has been done by many other researchers [1-4, 6, 14, 15 17, 18], and a great number of testers have been developed. Although those works opened a new era of understanding the mechanical properties of woven fabrics and designing the fabric 
tensile testers, it should be noted that because of the complex mechanical properties of woven fabrics, the existing testers which are theoretically capable of making the measurement are still unsuitable to be applied into the practical use or have serious limitations.

Among the methods for measuring the anisotropic tensile properties of woven fabrics that have been previously used, there are two main categories according to the test principle: measurement by the uniaxial test method, and measurement by the strip biaxial test method. The difference between the two methods is in that in the former, the transverse deformation of a sample is not restrained so that a simple shear occurs in the bias direction, whereas in the latter, the transverse deformation of a sample is restrained so that the shear deformation does not occur. With a conventionally anisotropic tensile test method, it is common to perform the tensile test with preparing a sample for various directions, such as the warp direction, the weft direction and the bias direction, and repeating the test for each sample. Unfortunately, not only it is a time-consuming, tedious process, but also the reliability and the reproducibility of the experiment results are very low because the initial condition is different. Furthermore, in the practical applications both in engineering and in apparel industry, there are a number of fabrics with no orthogonal symmetry, which are difficult to be theorized to deal with until now. It is highly desirable, therefore, to investigate the anisotropic tensile properties of woven fabrics in a simpler way.

In this study, a multi-axial circular tensile tester (MACTT) for measuring the anisotropic 
tensile properties of woven fabrics is developed. The new tester can be used to measure tensile modulus in various directions simultaneously for one sample. Then the relationship among the orthotropic tensile properties measured by the new test method, the uniaxial test and the strip biaxial test were also studied. The experiment results tested by the new tester with the theoretical results calculated with based on Hooke's Law were also compared . Finally, the experimental results by the new tester were compared with those by the strip biaxial tester for some special woven fabrics. The experimental results contribute to the conclusion that the new tester is not only theoretically reasonable but also practically feasible for measuring anisotropic tensile properties for small strain.

\section{Theoretical background}

Although the mechanical properties of woven fabrics are nonlinear in general, they may be regarded as linear elastic orthotropic material sheets in a numerical model when deformation is very small. For orthotropic materials, the relationship between stress and strain is in accordance with Hooke’s Law.

Figure 1 shows that a woven fabric is rotated by an angle $\theta$ from the $x$ and $y$ directions to the $X$ and $Y$ directions. Where $x$ axis is taken along the weft direction, $\mathrm{y}$ axis is taken along the warp direction, and $\theta$ is the axis rotation angle from the $x$ axis to the $X$ axis. 


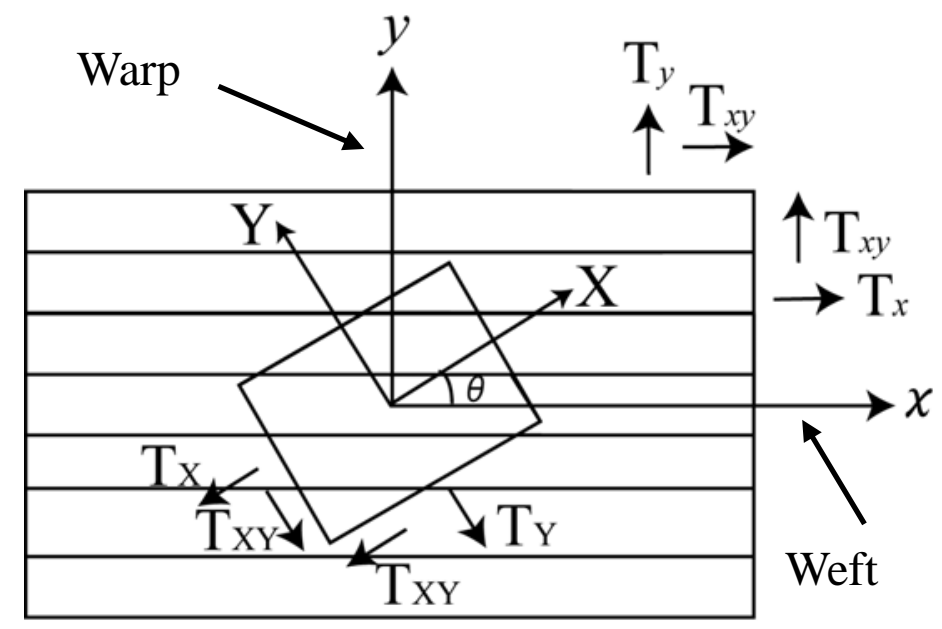

Figure 1. Rotation of arbitrary axes $(X Y)$ from fabric axes $(x y)$.

Applying transformation law of stress and strain using direction cosine, putting $l=\cos \theta$, $m=\sin \theta$, we can obtain the following expressions [7]:

For stress $T$ :

$$
\left[\begin{array}{l}
T_{x} \\
T_{y} \\
T_{x y}
\end{array}\right]=\left[\begin{array}{ccc}
l^{2} & m^{2} & -2 l m \\
m^{2} & l^{2} & 2 l m \\
l m & -l m & l^{2}-m^{2}
\end{array}\right]\left[\begin{array}{c}
T_{X} \\
T_{Y} \\
T_{X Y}
\end{array}\right]
$$

For strain $\varepsilon$ :

$$
\left[\begin{array}{c}
\varepsilon_{X} \\
\varepsilon_{Y} \\
\varepsilon_{X Y}
\end{array}\right]=\left[\begin{array}{ccc}
l^{2} & m^{2} & l m \\
m^{2} & l^{2} & -l m \\
-2 l m & 2 l m & l^{2}-m^{2}
\end{array}\right]\left[\begin{array}{c}
\varepsilon_{x} \\
\varepsilon_{y} \\
\varepsilon_{x y}
\end{array}\right]
$$

The relation between stress and strain in the $X, Y$ directions is expressed as follows using Hooke's law

$$
\left[\begin{array}{c}
\varepsilon_{X} \\
\varepsilon_{Y} \\
\varepsilon_{X Y}
\end{array}\right]=\left[\begin{array}{lll}
C_{X X} & C_{X Y} & C_{X S} \\
C_{X Y} & C_{Y Y} & C_{Y S} \\
C_{X S} & C_{Y S} & C_{S S}
\end{array}\right]\left[\begin{array}{c}
T_{X} \\
T_{Y} \\
T_{X Y}
\end{array}\right]
$$

Where 


$$
C_{X X}=\frac{l^{4}}{E_{x}}+\frac{m^{4}}{E_{y}}+l^{2} m^{2}\left(\frac{1}{G_{x y}}-2 \frac{\mu_{y}}{E_{x}}\right)
$$

Or

$$
\left[\begin{array}{l}
T_{X} \\
T_{Y} \\
T_{X Y}
\end{array}\right]=\left[\begin{array}{lll}
A_{X X} & A_{X Y} & A_{X S} \\
A_{Y X} & A_{Y Y} & A_{Y S} \\
A_{X S} & A_{Y S} & A_{S S}
\end{array}\right]\left[\begin{array}{c}
\varepsilon_{X} \\
\varepsilon_{Y} \\
\varepsilon_{X Y}
\end{array}\right]
$$

Where:

$$
A_{X X}=\frac{E_{x} l^{4}}{1-\mu_{x} \mu_{y}}+\frac{E_{y} m^{4}}{1-\mu_{x} \mu_{y}}+2 l^{2} m^{2}\left(\frac{E_{x} \mu_{y}}{1-\mu_{x} \mu_{y}}+2 G_{x y}\right)
$$

Where $E_{x}$ and $E_{y}$ are the tensile modulus in the $X$ and $Y$ directions, $\mu_{\mathrm{x}}$ or $\mu_{\mathrm{y}}$ is the apparent Poisson's ratio when a sample is tensioned along the $X$ or $Y$ direction, and $G_{x y}$ represents the shear modulus.

1. Theoretical model of the uniaxial test

In the case of the uniaxial test, a stress state is defined by setting $T_{Y}=0, T_{X Y}=0$ in the stress-strain relations given in Equation 3. From the Equation 3, we obtain:

$$
\begin{aligned}
\frac{\varepsilon_{X}}{T_{X}}=C_{X X}= & \frac{l^{4}}{E_{x}}+\frac{m^{4}}{E_{y}}+l^{2} m^{2}\left(\frac{1}{G_{x y}}-2 \frac{\mu_{y}}{E_{x}}\right) \\
& =\frac{1}{E_{x}} \cos ^{4} \theta+\frac{1}{E_{y}} \sin ^{4} \theta+\cos ^{2} \theta \sin ^{2} \theta\left(\frac{1}{G_{x y}}-2 \frac{\mu_{y}}{E_{x}}\right)
\end{aligned}
$$

2. Theoretical model of the strip biaxial test

In the case of the biaxial test, a strain state is defined by setting $\varepsilon_{X}=0, \varepsilon_{X Y}=0$ in the stress-strain relations given in equation 4. From the Equation 4, we obtain: 


$$
\begin{aligned}
\frac{T_{X}}{\varepsilon_{X}}=A_{X X}= & \frac{E_{x} l^{4}}{1-\mu_{x} \mu_{y}}+\frac{E_{y} m^{4}}{1-\mu_{x} \mu_{y}}+2 l^{2} m^{2}\left(\frac{E_{x 1} \mu_{y}}{1-\mu_{x} \mu_{y}}+2 G_{x y}\right) \\
= & \frac{E_{x}}{1-\mu_{x} \mu_{y}} \cos ^{4} \theta+\frac{E_{y}}{1-\mu_{x} \mu_{y}} \sin ^{4} \theta+2 \cos ^{2} \theta \sin ^{2} \theta\left(\frac{E_{x} \mu_{y}}{1-\mu_{x} \mu_{y}}+2 G_{x y}\right)
\end{aligned}
$$

3. Theoretical model of the multi-axial circular tensile test

Figure 2 (a) shows the tensile mode of a fabric sample used in the multi-axial circular tensile test.

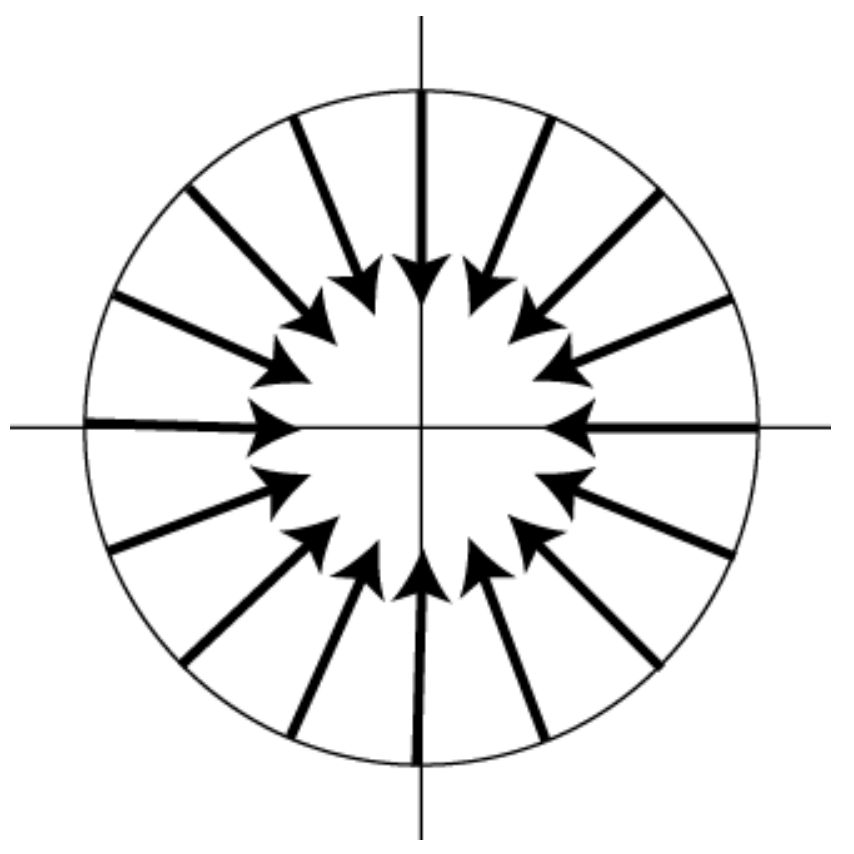

(a) Multi-axial circular tensile mode.

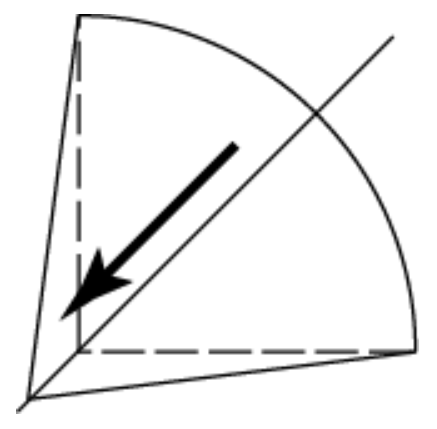

(b) A unit structure.

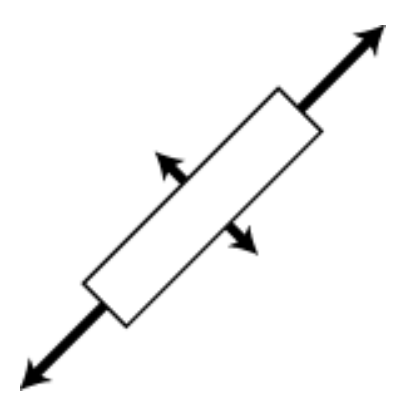

(c) Approximated tensile mode.

Figure 2. Tensile mode of the multi-axial circular tensile tester. 
In order to generate stresses on a sample in various directions simultaneously, a pressure is exerted on the center of the sample by a perpendicular load. As shown in Figure 2 (b) and (c), it is assumed that the tensile deformation mode in the multi-axial circular tensile test is analogous to that in the strip biaxial test, and the stress-strain theoretical model can be also expressed as Equation 6. By this way, it is easy to measure anisotropic tensile properties of woven fabrics with one sample simultaneously.

\section{Apparatus}

The new tester comprises four sections: a loading section, a measuring section, a clamping section and a control/memory section. 16 load sensors are installed in equal interval angle $22.5^{\circ}$ around a circumference of steel thick cylinder to measure the tensile loads in 16 directions.

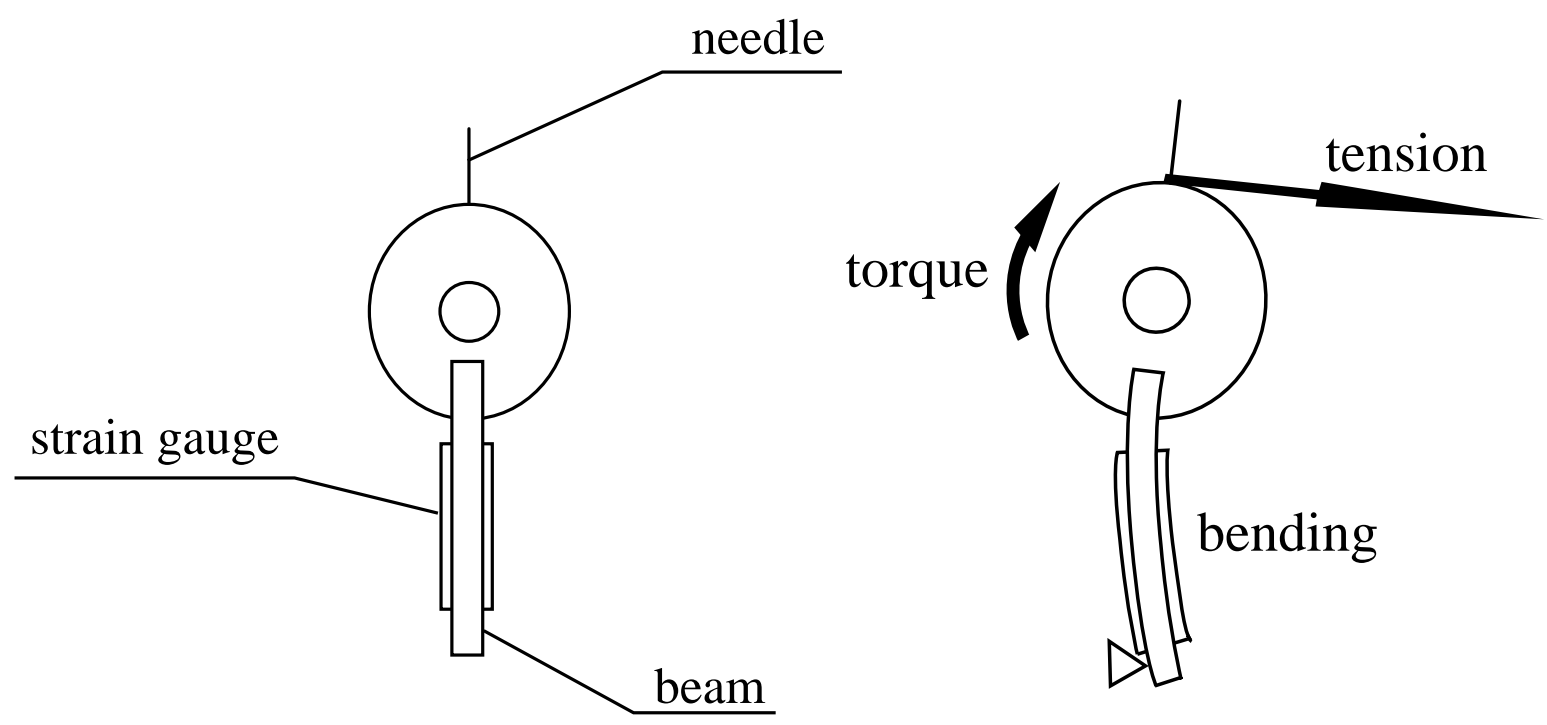

$\begin{array}{ll}\text { (a) Without tensile load. } & \text { (b) With tensile load. }\end{array}$

Figure 3. The sensor of the multi-axial circular tensile tester. 
The sensor is shown in Figure 3. This is a strain gauge sensor with needles on the top, which can be used to fix a sample. Each sensor is calibrated by actual load.

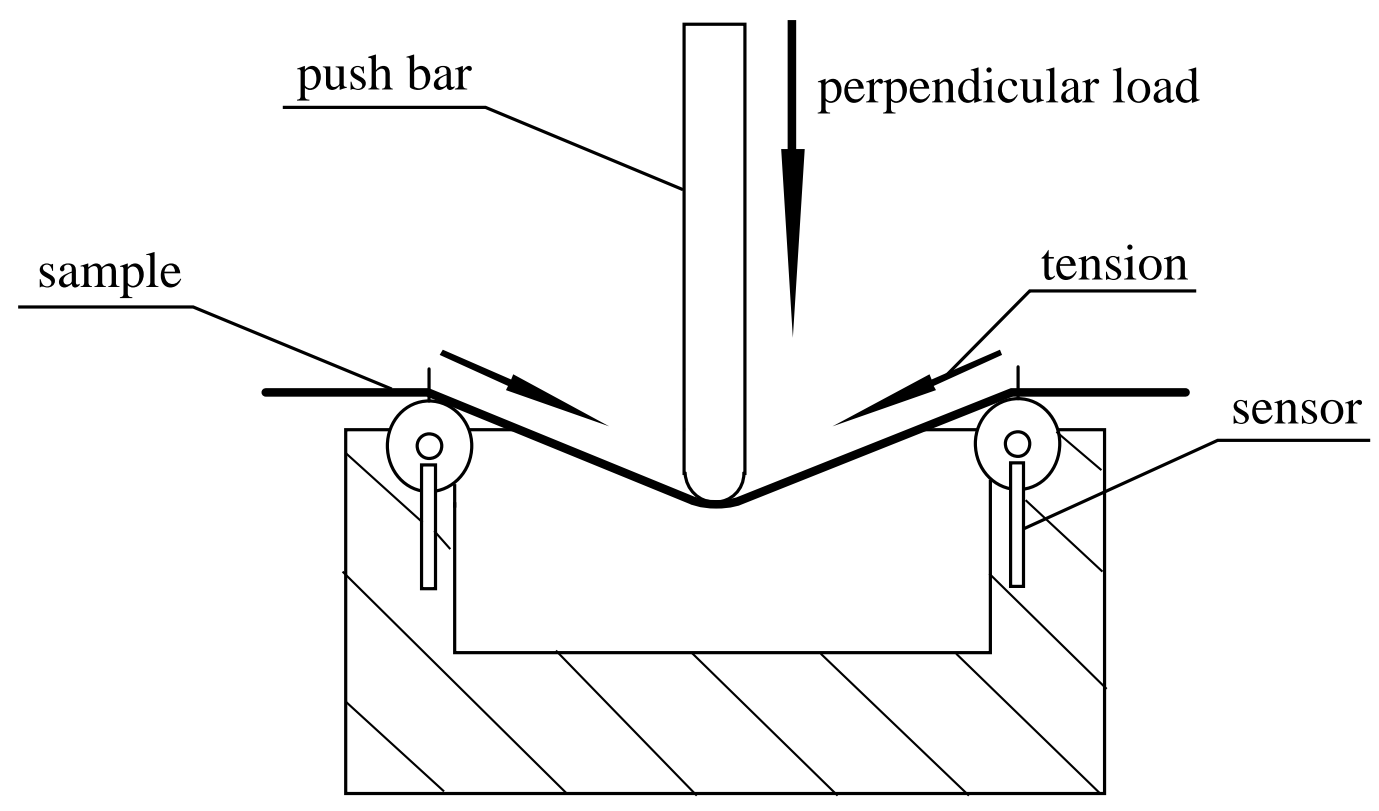

Figure 4. The loading section of the multi-axial circular tensile tester.

The loading section is shown in Figure 4. The diameter of circle which sensors were arranged is $140 \mathrm{~mm}$. The tip of the push rod is a hemisphere of diameter $20 \mathrm{~mm}$. The tensile load is acted on a sample by uniform movement of an electric slide rod exerting the perpendicular load on the center of a sample. With the load sensors, 16 tensile loads can be measured. The stress is assumed that the tensile load per unit length of $1 / 16$ sensor circumference. The perpendicular displacement can be measured by the movement of the rod. The maximal tensile deformation of the sample is assumed to be the change of diameter along the sample and can be obtained geometrically by the perpendicular displacement. The strain can be simplified as the average value that is the maximal tensile deformation divided 
by the number of sampling points because the inclined angle change for each sampling is so

little that it can be neglected. With a preload section, an initial tensile force can be applied to a sample to suppress misalignment. In a square frame, a sample is fixed on the adjacent two sides with two fixed bar magnets and the preload is applied to the other two sides with iron bars as weight. Through an A/D converter, the measured load and strain data are inputted into a personal computer for processing to obtain the stress-strain curve.

\section{Experiments}

6 kinds of fabric samples were selected. They are made with cotton, wool or polyester respectively and their weaves are plain, twill, rib and satin. Sample 1, sample 2 and sample 5 were selected to verify theoretical model of the new tester because of their orthotropy. Sample 3, sample 4 and sample 6 were selected to make a comparison between the KES-FB1 tester [9] and the new tester. Details of the samples are summarized in Table 1.

Table 1. Details of the samples.

\begin{tabular}{|c|c|c|c|c|c|c|c|c|c|c|}
\hline \multirow{2}{*}{ Sample } & Material & \multirow[t]{2}{*}{ Weave } & \multirow{2}{*}{$\begin{array}{l}\text { Mass } \\
\left(\mathrm{g} / \mathrm{m}^{2}\right)\end{array}$} & \multirow{2}{*}{$\begin{array}{l}\text { Thick } \\
\text { ness } \\
\text { (mm) }\end{array}$} & \multicolumn{2}{|c|}{$\begin{array}{l}\text { Fabric density } \\
\text { (Number / cm) }\end{array}$} & \multicolumn{2}{|c|}{$\begin{array}{l}\text { Yarn count } \\
\quad(\text { tex })\end{array}$} & \multicolumn{2}{|c|}{$\begin{array}{l}\text { Crimp } \\
(\%)\end{array}$} \\
\hline & Warp Weft & & & & Warp & Weft & Warp & Weft & Warp & Weft \\
\hline Sample & Cotton & Plain & 228.2 & 0.48 & 20.8 & 15.2 & 59.5 & 57.6 & 7.8 & 7.3 \\
\hline Sample & Wool & Plain & 160.4 & 0.31 & 20.0 & 21.2 & 31.7 & 39.6 & 5.8 & 7.3 \\
\hline Sample & Cotton & 3/1 Left Twill & 195.4 & 0.39 & 44.4 & 23.6 & 27 & 28.8 & 6.2 & 4.0 \\
\hline Sample & Wool & 2/2 Right Twills & 132.5 & 0.30 & 22.0 & 20.8 & 25.4 & 27.9 & 5.8 & 7.8 \\
\hline Sample & Cotton & Rib & 160.1 & 0.32 & 69.6 & 14.0 & 14.2 & 40.9 & 2.5 & 4 \\
\hline $\begin{array}{l}\text { Sample } \\
\end{array}$ & Polyester & 5 Harness Satins & 111.3 & 0.16 & 34.4 & 13.6 & 6.4 & 17.9 & 1.1 & 1.6 \\
\hline
\end{tabular}

To perform the uniaxial test and the strip biaxial test, the roller clamps method [2] and

KES tester are used to measure the anisotropic tensile properties of woven fabrics, 
respectively. Since the anisotropic tensile properties cannot be measured directly by these two methods, the samples are cut in the warp and weft directions and also in directions at $\pm 22.5^{0}, \pm 45^{0}, \pm 67.5^{0}, \pm 112.5^{0}, \pm 135^{0}, \pm 157.5^{0}$ to the warp. They were tested in the usual way.

\section{Results and Discussion}

1. Comparison of the stress distributions of orthotropic fabrics by three test methods.

Figure 5 illustrates the stress distribution of sample 1 by the three test methods when the strain is set as $1.2 \%$. Sample 2 and sample 5 showed the same tendency.

$\rightarrow$ Roller clamps

$\square$ MACTT

$\rightarrow$ KES
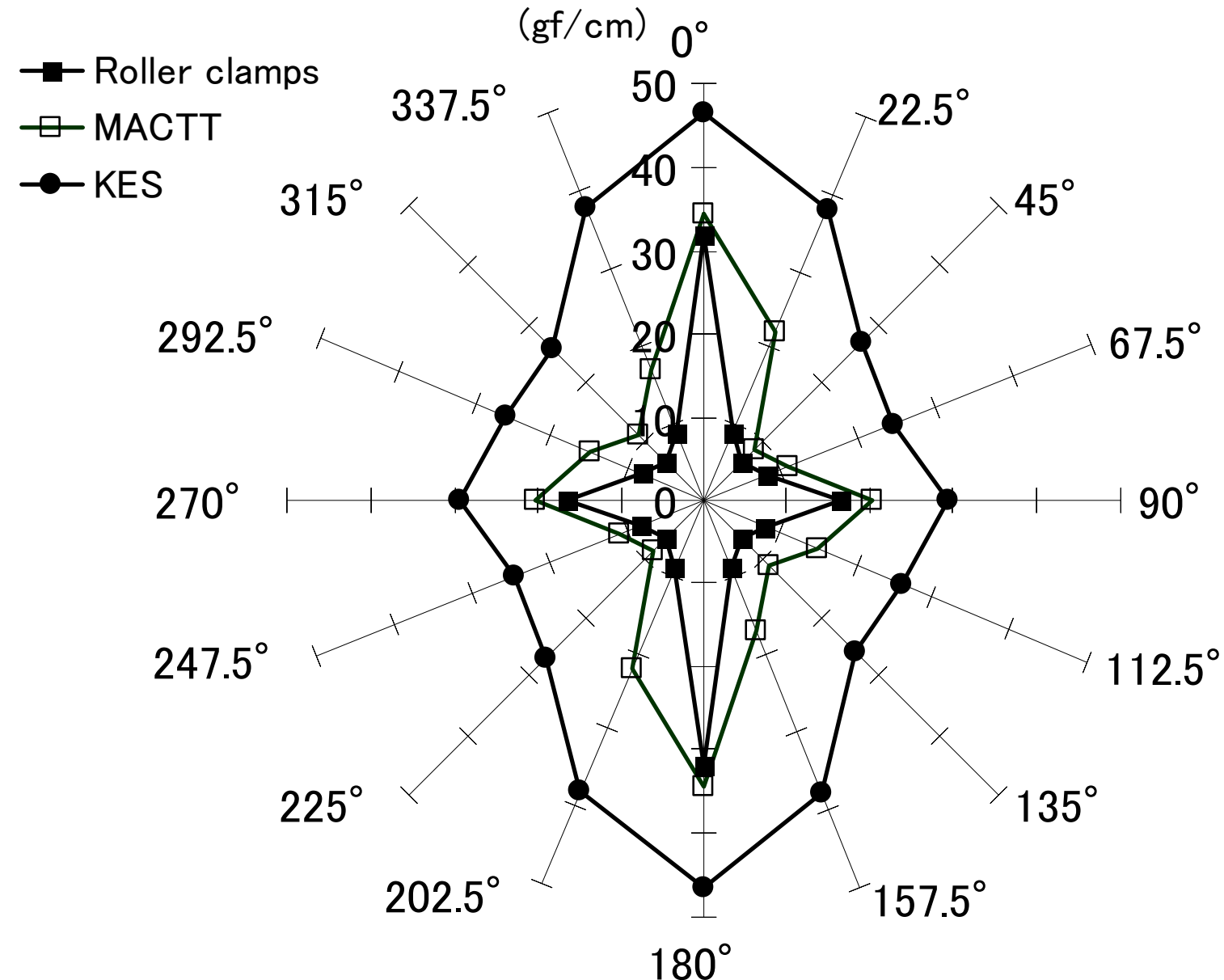

Figure 5. Stress distributions by three test methods. (Sample 1, strain $=1.2 \%$ ) 
In spite of the same sample and at the same strain, the three tensile stress distributions show a considerable difference. The reason is mainly due to the different clamping arrangements. For the KES tester, because the clamped sections are comparatively larger, the Poisson's effect and shear deformation are restrained, which will generate the largest loads and thus making orthotropic tensile property the least marked. On the contrary, for the roller clamps method, because there is no theoretical restraint, the transversal deformation is free, which will result in the smallest load, especially in the bias direction. For the new tester, the experiment results show that the shape of the tensile contribution is nearly similar to that obtained by the KES tester. This truth proves that the theoretical hypothesis on the tensile deformation mode in the new test is reliable. Although these two deformation modes are approximated to the strip biaxial deformation, a sample used in the new tester is gripped at 16 points by the needles so that it can be considered that there is a loading escape occurring on the gripping points. As a result, the deformation restraint of the sample in the multi-axial tensile test is not so severe as that in the KES test. Therefore, as shown in Figure 5, for the same sample, the stress in the same direction measured by the new tester is smaller than that measured by the KES tester, and larger than that measured by the roller clamps method, but the difference is little. So the orthotropic tensile distribution by the new tester shows more significant.

2. Comparison of the experiment results by the new tester and those by the orthotropic theory. 
As mentioned before, according to the Hooke's Law, we obtain the theoretical model of the new tester as shown in Equation 6. To calculate the theoretical curve, we set:

$$
\frac{E_{x}}{1-\mu_{x} \mu_{y}}=E_{1}^{\prime}, \quad \frac{E_{y}}{1-\mu_{x} \mu_{y}}=E_{2}^{\prime}, \quad 2\left(\frac{E_{1} \mu_{y}}{1-\mu_{x} \mu_{y}}+2 G_{x y}\right)=k
$$

So that Equation 6 can be expressed as Equation 7:

$$
\frac{T_{X}}{\varepsilon_{X}}=E_{1}^{\prime} \cos ^{4} \theta+k \cos ^{2} \theta \sin ^{2} \theta+E_{2}^{\prime} \sin ^{4} \theta
$$

From Equation 7, using the experiment results: $T 0^{\circ}=34.4 \mathrm{gf} / \mathrm{cm}, T 45^{\circ}=8.5 \mathrm{gf} / \mathrm{cm}, T 90^{\circ}=$ 20.2gf/cm, and $\varepsilon=0.012 \mathrm{~cm}$, which are measured by the new tester, the theoretically approximate curve can be obtained.

Figure 6 shows the measured stress distribution curve of sample 1 by the new tester and the theoretically approximate stress distribution curve was calculated with base on the Hooke's Law.

- theoretical curve - measured data (MACTT)

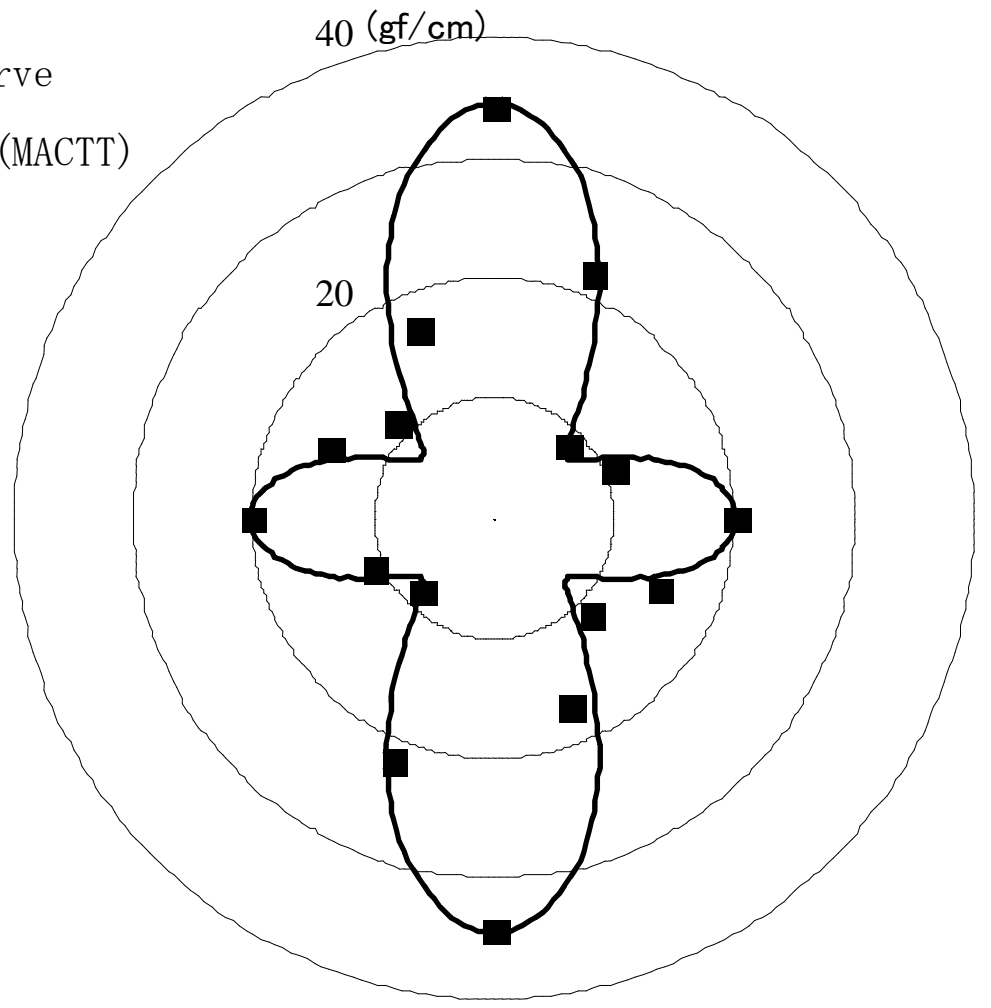


Figure 6. The measured stress distribution and theoretically approximate curve. (Sample 1, strain $=1.2 \%)$

It can be seen a good agreement between the two results when the strain is small. Consequently, it can be concluded that in the case of orthotropic fabrics, tensile properties in the arbitrary directions can be estimated only from three experiment values in the following three directions: the warp direction, the weft direction and the $45^{\circ}$ bias direction, respectively.

3. Comparison of the experiment results of unorthotropic fabrics by the new tester and those by the KES tester

Figure $7 \mathrm{a}$ and Figure $7 \mathrm{~b}$ show the stress distributions of sample 3 measured by the new tester and the KES tester, respectively.

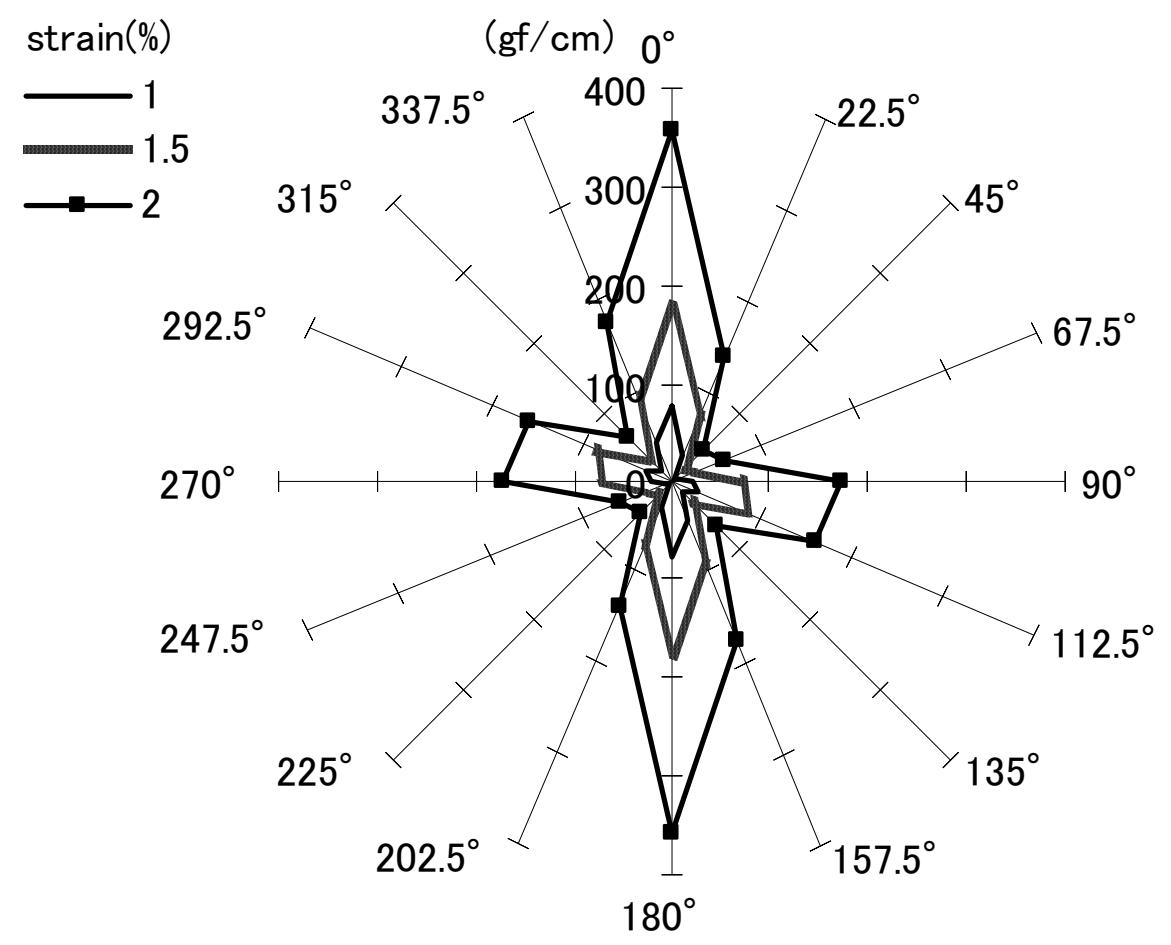


Figure 7a. Stress distributions of Sample3 (MACTT).

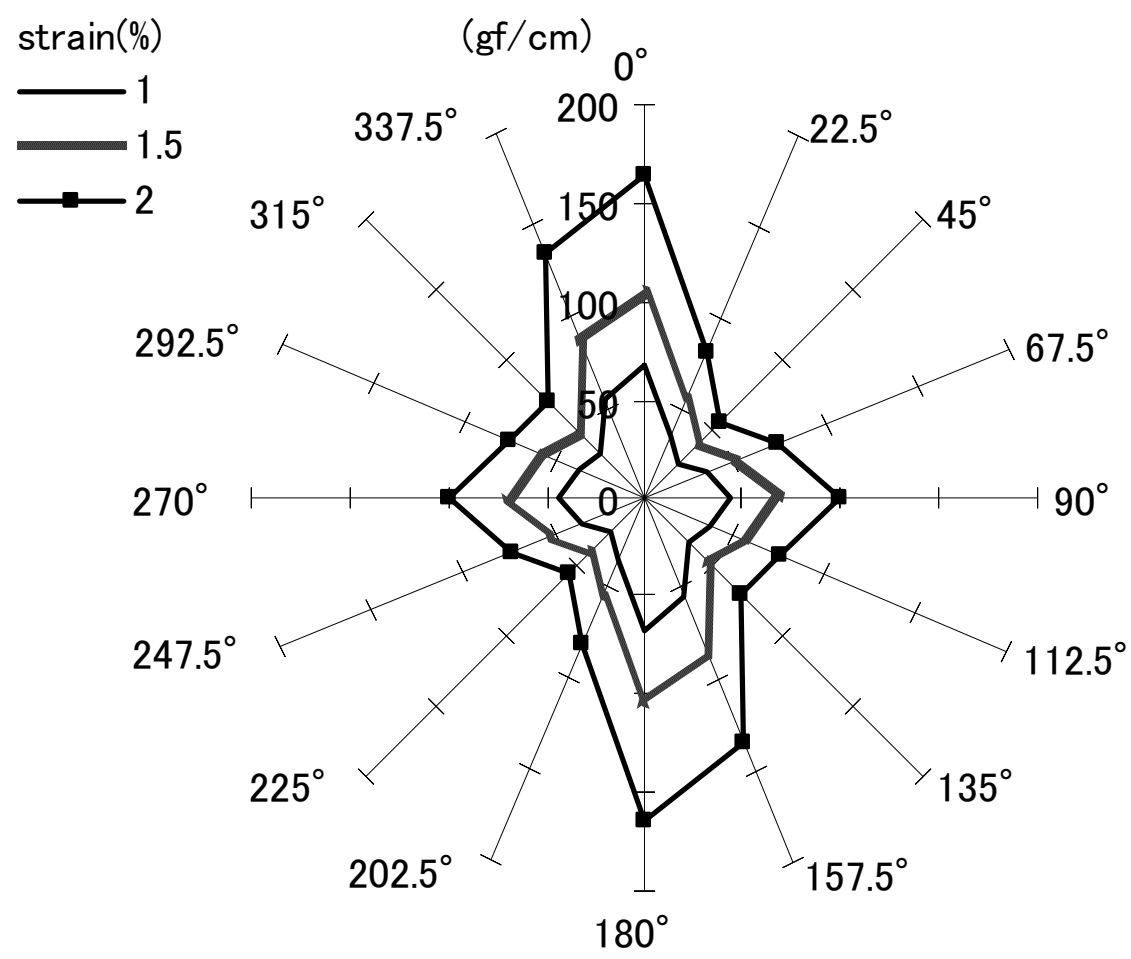

Figure 7b. Stress distributions of Sample3 (KES).

Both of the experiment results indicate that sample 3 exhibits not orthotropic but complicated shape in the stress distributions. The stress distributions are symmetrical about the origin of coordinate and larger load values show in the direction of the twill line. This is attributed to the fact that sample 3 is a cotton 3/1 left twills, which is an obviously non-symmetric structure from the viewpoint of the fabric weave. It is considered that shear deformation will occur when twill fabrics are carried out tensile deformation along a yarn direction, and this phenomenon will have an effect on the anisotropic distributions. Moreover, 
for twill fabrics, the warp yarns may be not orthogonal to the weft yarns because of its especial structure, and it may also been thought to influence the anisotropic distributions.

Figure $8 \mathrm{a}$ and Figure $8 \mathrm{~b}$ show the stress distributions of sample 4 measured by the new tester and the KES tester, respectively.

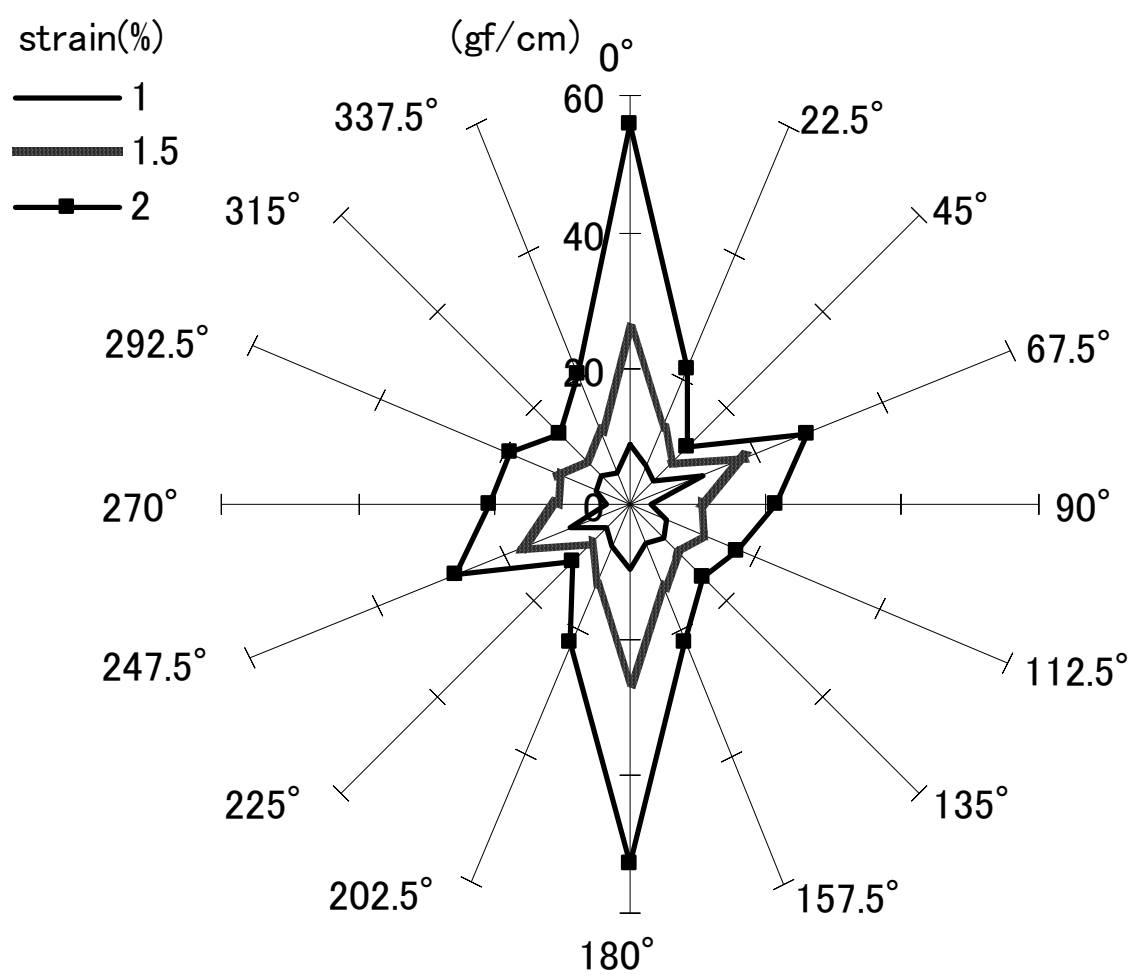

Figure 8a. Stress distributions of Sample4 (MACTT).

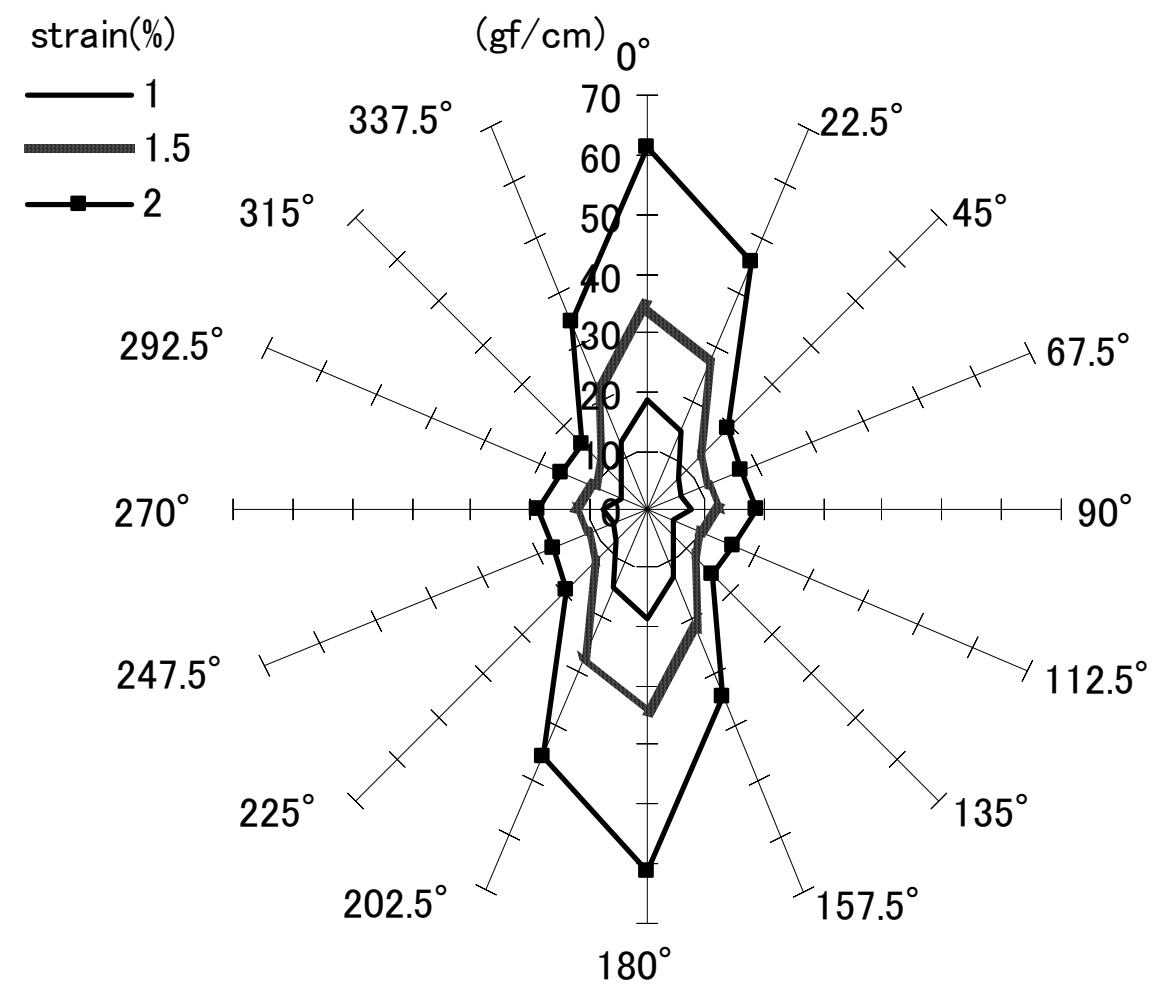


Figure 8b. Stress distributions of Sample4 (KES).

Like sample 3, both of the experiment results indicate that sample 4 exhibits not orthotropic but complicated shape in the stress distributions. The stress distributions are symmetrical about the origin of coordinate and larger load values show in the direction of the twill line. It can be explained by the same reasons as sample 3 .

In such the cases, it is difficult to predict actual stress distribution in advance. Therefore, it is especially more useful and easier to measure anisotropic tensile properties with the new tester.

Figure 9a and Figure 9b show the stress distributions of sample 6 measured by the new tester and the KES tester, respectively.

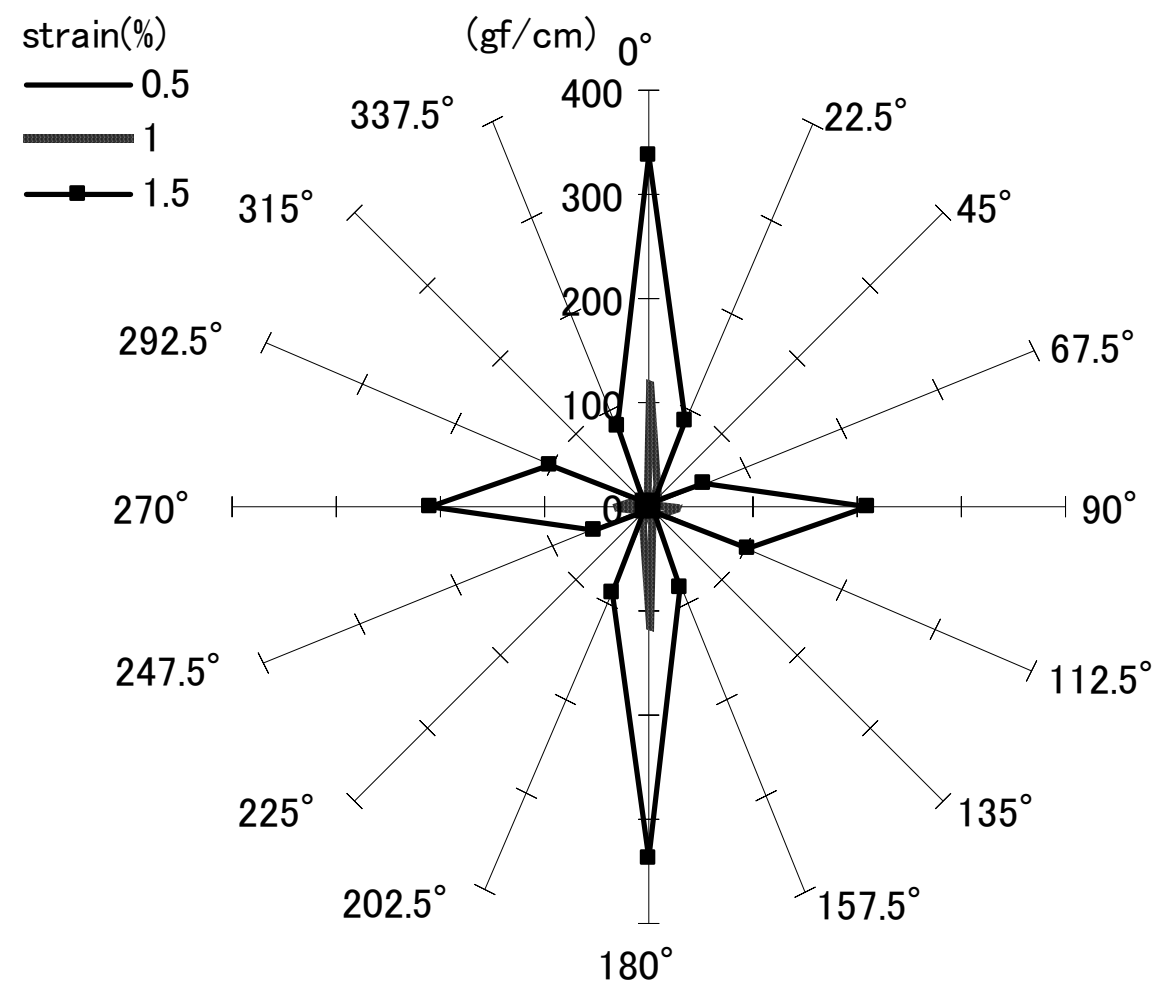


Figure 9a. Stress distributions of Sample6 (MACTT).

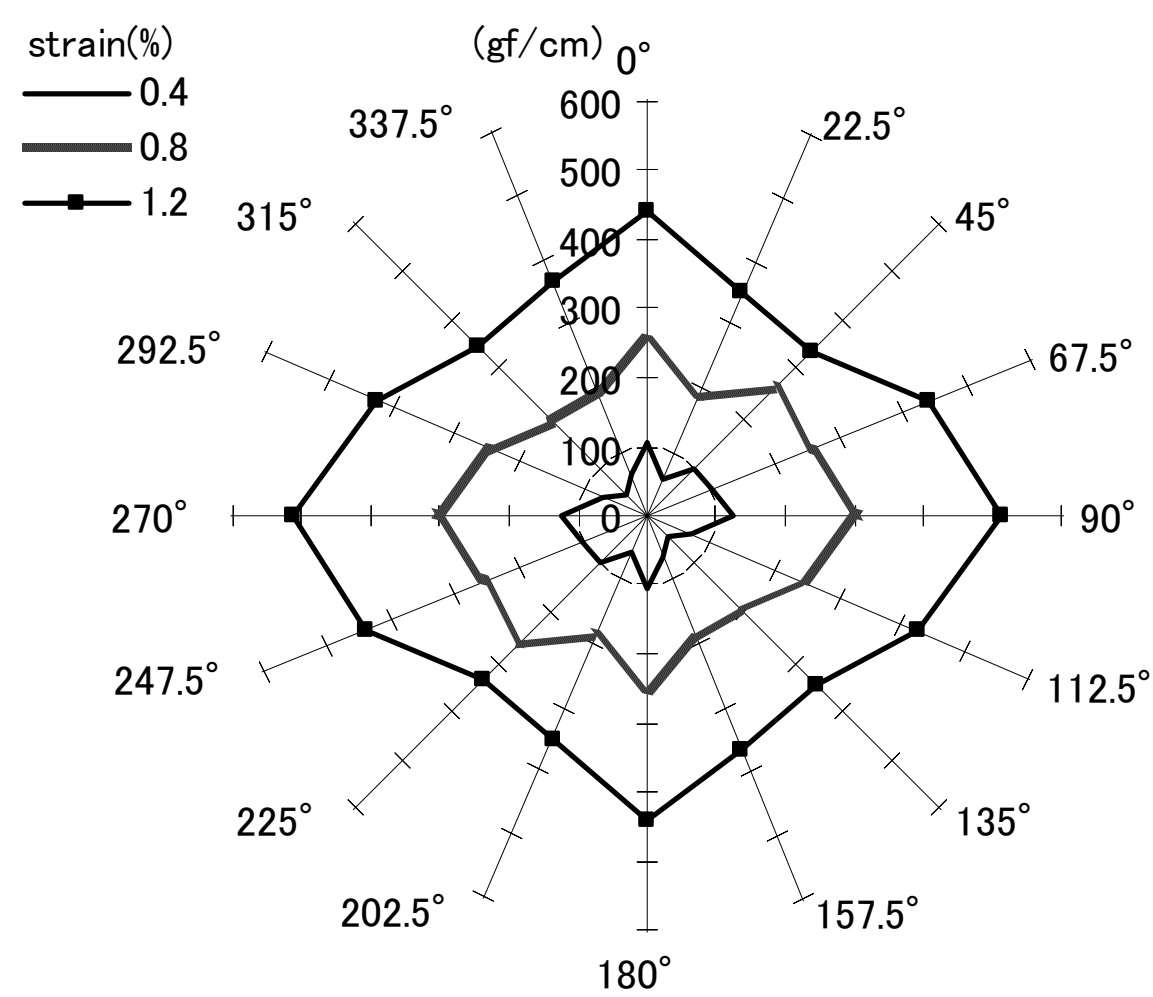

Figure 9b. Stress distributions of Sample6 (KES).

There is a big difference between these two experiment results. For the new tester, the larger load values show in the warp direction and the weft direction and there is few load in the bias directions. For the KES tester, the stress distributions behave like circular forms. It can be explained as follows: because sample 6 is made of smooth polyester filament yarns with 5 harness satins, few interlacing point results in shear deformation occurring easily. The new tester gives little effect on the shear deformation, so the stress distributions show good orthotropic properties. On the other hand, the KES tester restricts the change of the interlace 
angle of yarns because of its clamping section. As a result, larger loads are generated in the bias directions in order to exert all tensile loads to pull the yarns to prevent shear deformation. So the stress distributions show no orthotropic properties. Sample 6, rib weave, showed the same anisotropy as the plain weave of sample 1.

\section{Conclusion}

The mechanical deformation theory and some measurement principles of the multi-axial circular tensile tester are confirmed by comparing the experiment results of plain fabrics by the new tester with those by the other two test methods. The experiment values show good agreement with the theoretically approximate values. It is reliable that the tensile deformation mode of the multi-axial circular tensile tester is considered to be analogous to the strip biaxial tensile deformation.

For orthotropic fabrics, the tensile stress results by the new tester are nearly equal to those by the roller clamps method, and it is possible to estimate theoretically tensile properties in other directions with base on the tested results in the directions of $0^{\circ}, 45^{\circ}$ and $90^{\circ}$. So the orthotropic tensile distribution by the new tester shows more significant results.

For general fabrics, the anisotropic tensile properties can be measured easily with the new tester although it is difficult to understand their structures and mechanical characteristics systematically. So the new tester shows more important results.

By the circular multi-axial tensile tester, not only an error can be reduced because of the 
same initial conditions, but also the experimental samples and time can be saved, so it can be expected that the new tester will be used for evaluating the anisotropic tensile property of various fabrics. Due to the mechanism of the apparatus, the range of strain is small. In practical use for the fabrics that the property in large strain range is required, like knit, the improvement of apparatus will be necessary.

\section{Acknowledgements}

This research was partially supported by the Ministry of Education, Science, Sports and

Culture, Grant-in-Aid for 21st Century COE Program and Global COE Program. The authors thank to Mr. Yusuke Abe for his cooperation.

\section{References}

1. Bao, L., Takatera, M., and Shinohara, A., Error Evaluation in Measuring the Apparent Possion’s Ratios of Textile Fabrics by Uniaxial Tensile Test, Sen’i Gakkaishi, 53(1), 20-26 (1997).

2. Bao, L., Takatera, M., Shinohara, A., and Yamashita, S., Determining the Apparent Shear Rigidity of Textile Fabrics by Uniaxial Tensile Test, Sen'i Gakkaishi, 53(4), 139-145 (1997).

3. Bassett, R., J., Postle, R., and Pan N., Experiment Methods for Measuring Fabric Mechanical Properties: A Review and Analysis, Textile Res. J., 69, 866-875 (1999). 
4. Buckenham, P., Bias-extension Measurements on Woven Fabrics, J. Textile Inst., 88, 33 40 (1997).

5. Chadwick, G. E., Shorter, S. A., and Weissenberg, K., A Trellis Model for the Application and Study of Simple Pulls in Textile Materials, J. Textile Inst., 40, T111-T160 (1949).

6. Checkland, P. B., Bull, T.H., and Bakker, E. J., A Two-dimensional Load-extension Tester for Fabrics and Film, Textile Res. J., 28, 399-403 (1958).

7. Jones, R., M., “Mechanics of Composite Materials”, Taylor \&Francis, pp. 31-57, 1975.

8. Kageyama, M., Kawabata, S., and Niwa, M., H., The Validity of a "Linearizing Method" for Predicting the Biaxial-extension Properties of Fabrics, J. Textile Inst., 79, 543-567 (1988).

9. KATO TECH CO., LTD., The KES-FB1 Tensile-Shear Tester Instruction Manual.

10. Kawabata, S., Niwa, M. and Kawai, H., The Finite-deformation Theory of Plain-weave Fabrics, Part I : the Biaxial-Deformation Theory, J. Textile Inst., 64, 21-46 (1973).

11. Kawabata, S., Niwa, M. and Kawai, H., The Finite-deformation Theory of Plain-weave Fabrics, Part II : the Uniaxial-Deformation Theory, J. Textile Inst., 64, 47-61 (1973).

12. Kawabata, S., Niwa, M. and Kawai, H., The Finite-deformation Theory of Plain-weave Fabrics, Part III: the Shear Deformation Theory, J. Textile Inst., 64, $62-85$ (1973).

13. Kilby, W. F., 2-Planar Stress-strain Relationships in Woven Fabrics, J. Textile Inst., 54, T9-T27 (1962). 
14. Klein, W. G., Stress-strain Response of Fabrics under Two-dimensional Loading, Part I : The FRL Biaxial Tester, Textile Res. J., 29, 816-821 (1959).

15. Lloyd, D. W., and Hearle, J. W. S., An Examination of a "Wide-Jaw" Test for the Determination of Fabric Poisson Ratio, J. Textile Inst., 68, 299-302 (1977).

16. Peirce, F. T., Cloth Structure: Geometry, J. Textile Inst., 28, T45-T96 (1937).

17. Shanahan, W., J., Lloyd, D., W., and Hearle, J., W., S., Characterizing the Elastic Behavior of Textile Fabrics in Complex Deformations, Textile Res. J., 48, 495-505 (1978).

18. Takatera, M., Kumoda, N., Bao, L., Shimizu, Y., Kamijo, M., Hosoya, S., and Iida, H., Large Deformation of Woven Fabrics under Free Shearing Uniaxial Load, Sen'i Gakkaishi, 55(7), 306-314 (1999).

19. Weissenberg, K., The Use of a Trellis Model in the Mechanics of Homogeneous Materials, J. Textile Inst., 40, T89-T110 (1949). 